\title{
EASING THE CHEMISTRY BOTTLENECK: CAREERS IN HIGH-THROUGHPUT CHEMISTRY
}

Big investments
in technologies
such as high-
throughput
chemistry have
presented new
opportunities
and challenges,
but have also
highlighted the
question of
whether further
skills are
required to
implement
these new
technologies.

Ian Marsh, Library Design and Production Group, Sandwich Laboratories, Pfizer Limited, Sandwich, Kent CT13 9NJ, UK. e-mail:ianmarsh@ sandwich.pfizer.com
The drive to increase the speed and efficiency of drug discovery, from hit identification all the way through to the creation of development candidates has seen huge investments by major pharmaceutical companies in many new technologies, with the primary aim of synthesizing more compounds and screening them faster; all at reduced cost per compound or assay.

From the chemist's perspective, high-throughput and combinatorial chemistry has the greatest potential to affect drug discovery as part of the 'closed-loop' process, in which numerous compounds are selected for synthesis from a 'virtual library' of compounds that could be made by parallel chemistry methods. These compounds are then screened, active compounds are identified, and these are followed up using high-throughput chemistry from the same or related virtual libraries. The process is repeated, and improved active compounds are followed up again, using computational methods to aid compound selection, in further loops until the desired compound properties have been achieved.

Many university courses contain combinatorial chemistry in their curricula, and numerous academic groups are at the forefront of developing new chemistry and techniques for high-throughput synthesis, equipping people with a sound knowledge base. But what further skills are required to implement these chemical technologies in the pharmaceutical industry?

Fundamentally, chemistry does not change, whether the synthesis of a compound is carried out individually in a round-bottomed flask, or as a member of an array of one thousand compounds. However, for a chemist working in the field of high-throughput synthesis, there are undeniably substantial differences in designing and synthesizing arrays of hundreds, or even thousands, of compounds compared with preparing a single compound.

The differences are manifested particularly in the application of automation to synthesis - a high-throughput chemistry laboratory looks 'different', and might contain various robotics for liquid handling, reaction work-up, purification and analysis. This indicates that a background in analytical chemistry or engineering might be as important as synthetic chemistry. The dependence on automation requires key specialists in teams to develop, maintain and research new laboratory technologies, but for the chemist, an aptitude and appetite for applying automation to make compounds is the main requisite, rather than acutely honed engineering skills. Nevertheless, the skill level that is required to synthesize 1,000 pure compounds in a single library is considerable, and should not be underestimated.

A chemist developing new methodology for highthroughput synthesis needs to consider how a reaction sequence could be used to make ten or a hundred compounds, rather than a single compound, as the method that is used to make compounds is as valuable as the final products. Routes must allow the preparation of as wide a variety of target molecules as possible, while being safe, high yielding and efficient in minimizing both the number of steps and reagents used. In this respect, highthroughput chemistry has some similarity to process research and development, in which the aim is to develop scalable routes to a single target. Irrespective of whether the final aim is to prepare one kilogram of one compound or one milligram of one thousand individual compounds, the core skills that are required remain the same - a thorough knowledge of chemical transformations, reagents and their application.

The rise of technology also requires an increasing dependence on information technology (IT) skills. Managing the data flow for hundreds of compounds presents a major challenge, and becomes impossible without specialist desktop software to track the large volumes of data that are generated in the synthesis, purification and characterization of numerous compounds. Consequently, IT skills are an important part of high-throughput synthesis.

The design of target compounds in medicinal chemistry is of primary importance, and applies equally to the application of high-throughput synthesis in medicinal chemistry. Computational methods used to aid medicinal chemistry are of crucial importance in high-throughput medicinal chemistry. The design of large libraries made for enriching screening files might be targeted to gene or receptor families by chemotype, and be designed to show drug-like physical properties. Similarly, high-throughput chemistry can be used to probe structure-activity relationships rapidly, or be targeted towards preparing inhibitors of a specific enzyme, using guidance from tools such as structure-aided drug design. One of the most gratifying aspects of parallel synthesis is that, by enhancing productivity through making large numbers of compounds, biological screening provides a similarly large volume of data to drive target understanding forwards more rapidly.

Drug discovery is a multidisciplinary business, in which interaction between colleagues with a range of skills is crucial for success. High-throughput synthesis has an increasingly important role in drug discovery, and represents an exciting and challenging addition to career options for synthetic chemists at all levels. It is another medicinal chemistry discipline - a powerful tool available to chemists that can expedite drug discovery when used appropriately.

Ian Marsh 